\title{
Constant Strain Frequency Sweep Measurements on Granite Rock
}

\author{
Kristian C. E. Haller and Claes M. Hedberg \\ Blekinge Institute of Technology 37179 Karlskrona, Sweden \\ (Received 28 September 2007; published 13 February 2008)
}

\begin{abstract}
Like many materials, granite exhibits both nonlinear acoustic distortion and slow nonequilibrium dynamics. Measurements to date have shown a response from both phenomena simultaneously, thus crosscontaminating the results. In this Letter, constant strain frequency sweep measurements eliminate the slow dynamics and, for the first time, permit evaluation of nonlinearity by itself characterized by lower resonance frequencies and a steeper slope. Measurements such as these are necessary for the fundamental understanding of material dynamics, and for the creation and validation of descriptive models.
\end{abstract}

DOI: 10.1103/PhysRevLett.100.068501

PACS numbers: 91.60.Lj, 43.25.+y, 62.40.+i, 62.65.+k

The acoustic resonance frequency in granite rock decreases with an increase in strain due to a change in sound velocity; it exhibits resonance frequency peak bending. This is a display of a strong nonlinearity which is connected to material imperfections [1]. Measurements on rocks so far have been explained by nonlinear effects for strains below certain values, for example $5 \times 10^{-7}$ for Berea and $2 \times 10^{-7}$ for Fontainbleau sandstones [2]. At higher strains, the nonlinearity has been accompanied by a conditioning, assumed to be related to Slow Dynamics (SD) [3-5]. The concept of Slow Dynamics [6] is the decrease in sound velocity to a thermodynamic disturbance, and the following slow recovery towards the equilibrium. The nonlinear distortion and the slow nonequilibrium dynamics are related and normally take place simultaneously [7]. The only exception is a test on rubber which showed a large Slow Dynamics effect, while the nonlinear distortion was very low [8]. In comparison, an undamaged steel bar gives a comparatively negligible nonlinear response and no discernible slow dynamic effect.

A normal frequency sweep measurement has constant input force, and the response amplitude in the object changes with frequency, see Figs. 1 and 2(a). It spans over different strain levels which influences the thermodynamic state. In Fig. 1(a), the increasing frequency sweeps (solid lines) follow a different path than the subsequent decreasing frequency sweeps (dashed lines) even when having the same input amplitudes. Different sweep rates give different responses; the measurements are sweep rate dependent $[6,9,10]$. Resonance frequency peak bending is observed. In Fig. 1(b), the decreasing ratio between the output amplitude and the drive amplitude is showing the amplitude dependent attenuation.

The true mechanisms behind this are not fully understood. Advancement of this area benefit the fundamental material physics, as well as the more application related material characterization within geology and industry.

The nonlinear distortion and the slow nonequilibrium dynamics are interconnected phenomena. Pasqualini et al. described in a recent article the consequences of this as: "It is difficult, but essential, to disentangle conditioning or nonequilibrium and nonlinear effects. New experimental strategies have to be developed for this endeavor" [2]. By keeping the internal strain constant throughout the sweep, as presented in this Letter, the thermodynamic state is steady and effects of fast and slow dynamics can be separated, providing an improved base for the advancement of material dynamics theory.

The parameter of interest in a normal Constant input Force Sweep (CFS) is the wave field in the object which varies depending on its resonant properties. The resonance frequency is when the wave field has a maximum, see Fig. 2(a).

In contrast, in the Constant Strain Sweep (CSS), the internal strain is kept equal (the output strain amplitude is constant) by varying the input force. The parameter of interest is then this input force, which has a minimum at resonance, see Fig. 2(b). The point of this Letter is that this test will minimize the Slow Dynamic interference.

In a Letter by Guyer et al. [11], experiments were performed under a type of constant strain, but the time dependent conditioning effect that makes the time history of the strain field influence the temporary state of the material was not fully taken into account.

The test object in this Letter was a granite rock bar, $420 \mathrm{~mm}$ long and $50 \mathrm{~mm}$ in diameter. It was hanging in two fishing lines to create a free-free boundary condition and kept in a climate chamber with constant temperature and humidity so that these would not alter the thermodynamic equilibrium. The test configuration is shown in Fig. 3.

A longitudinal wave was generated at one end of the granite bar by a glued piezo transducer of $30 \mathrm{~mm}$ diameter and $2.5 \mathrm{~mm}$ thickness with a brass backload. The transducer was fed an electrical signal from an Agilent 33250A signal generator that was amplified by a Krohn-Hite 7500 amplifier and impedance matched by a Krohn-Hite MT75R transformer. The wave was picked up at the other end of the bar by a PCB 35C22 accelerometer, and the signal was processed by a Stanford Research SRS830 lock-in amplifier. Before the start of measurements, the sample hung for 


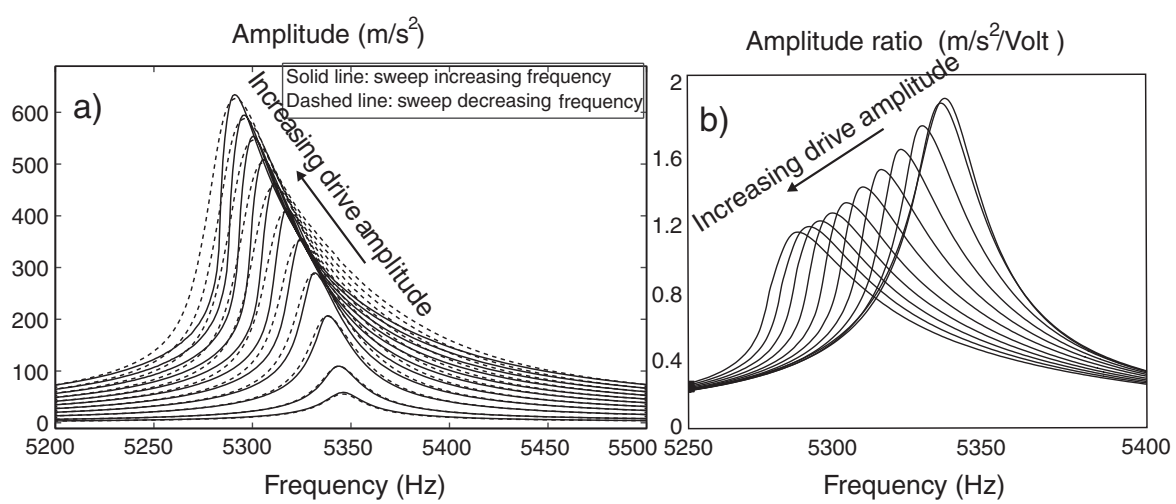

FIG. 1. In (a), normal frequency sweep, which measures the output response amplitude for a constant input force. The resonance frequency shift and the resonance frequency shift bending are present. In (b), the ratio between the output amplitude and the input amplitude indicates an amplitude dependent attenuation — increasing with amplitude.

two weeks resting in the climate chamber at test temperature $20 \pm 0.05{ }^{\circ} \mathrm{C}$ and test relative humidity $50 \pm 0.1 \%$, so that an equilibrium state was reached.

The strain can be calculated from $\epsilon=a /\left(\omega^{2} L\right)$, where $\epsilon$ is strain, $a$ is acceleration, $\omega$ is angular velocity, and $L$ is the length of the sample. When conducting the CSS protocol, it is important to not exceed the strain level. Therefore, for every new frequency step, the excitation force is decreased to about 5\% less than the estimated target value. Thereafter, it is slowly increased to the strain target value within an error margin of $+0 \%$ and $-1 \%$. This procedure is time consuming - a typical sweep rate is 5 minutes $/ \mathrm{Hz}$ - but allows the object to stay close to its thermodynamic equilibrium during the measurement. The strain schemes as function of time are seen in Fig. 4. The frequency is increased in steps of $1 \mathrm{~Hz}$.

The smooth curve represents the CFS measurement, and the square curve represents the CSS measurement. For the CFS, the strain at resonance (the maxima of the smooth a) Constant Force Sweep
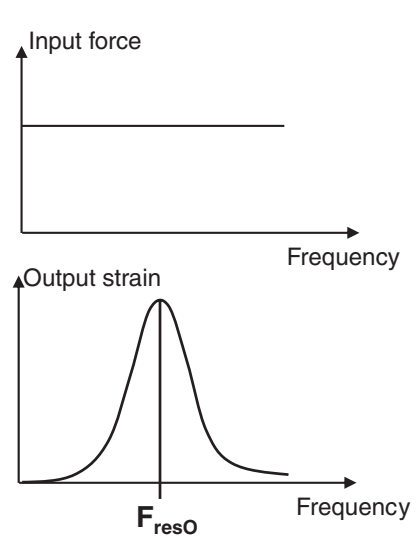

b) Constant Strain Sweep
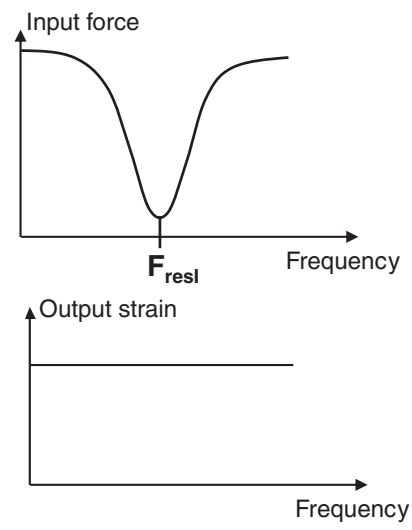

FIG. 2. In (a), the input force and the output strain for a normal frequency sweep. In (b), the input force and the output strain for a constant strain frequency sweep. curves) is the same as the constant level in the constant strain scheme (CSS - the rectangular curve). The scheme switches between a low level which is always the same, and a higher level which increases for every new frequency sweep which starts at frequency $f_{0}$ and ends at frequency $f_{1}$. Even though the measurement are made at a slow speed $-5 \mathrm{sec} / \mathrm{Hz}$ sweep rate for the CFS and between 0.5-6 minutes $/ \mathrm{Hz}$ for the CSS - the low strain state is not at equilibrium for any of the protocols because a recovery process takes place during the measurements, in particular, going from the high to the low strain level. The CFS protocol constantly changes the strain level during the measurement, and therefore the thermodynamic state of the material is never stable. As a contrast, the square constant strain curve in Fig. 4 allows the material to stay close to its thermodynamic equilibrium during each interval $\Delta t$ of the high strains.

Frequency sweep curves using the normal CFS technique are found in Fig. 5 where the resonance frequencies shift with increase in excitation amplitude - peak bending is observed. The curves in Fig. 5(a) are with increasing input amplitude ("high"). Figure 5(b) consists of all the low strain sweeps, more or less on top of each other. The peaks are plotted in Fig. 6 with respect to the maximum high strain. The high amplitude peaks are plotted in

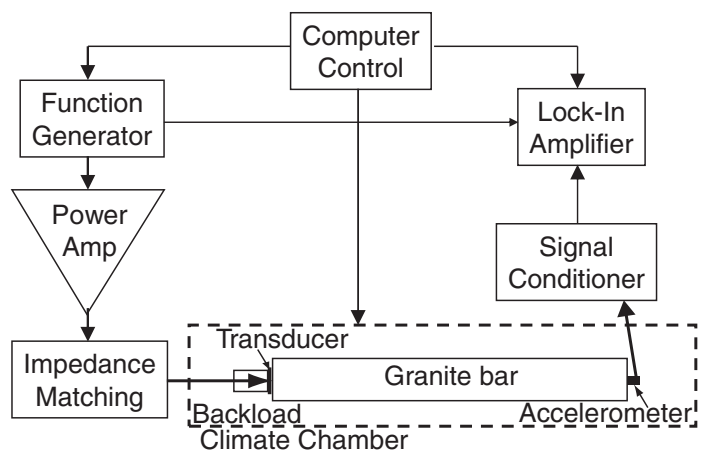

FIG. 3. Test configuration. 


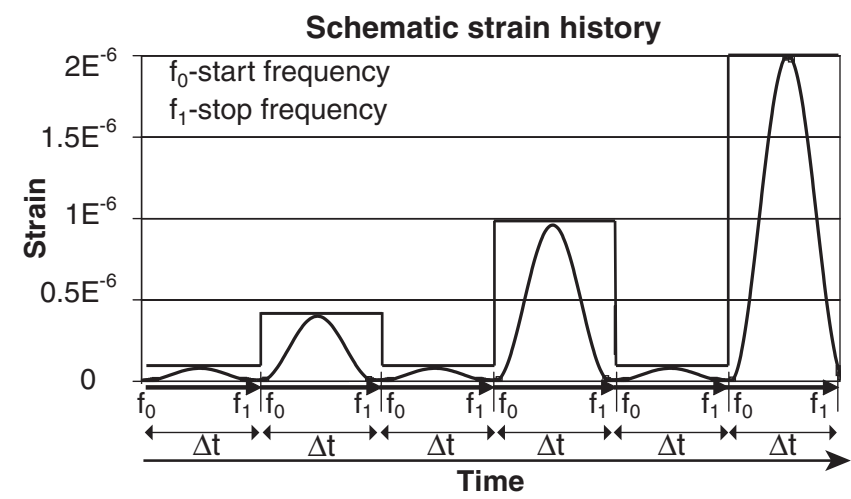

FIG. 4. The strain history. The smooth curve in Fig. 4 is the strain of the normal sweep (CFS), and the rectangular curve is the strain for the constant strain sweep (CSS).

Fig. 6(a), and the low control amplitude peaks are plotted in Fig. 6(b). The high amplitude sweeps early show a nonlinear frequency shift behavior. It is hard to say exactly where it starts, possibly at $1 \times 10^{-7}$. In earlier work, a threshold $\epsilon_{L}$ between linear and nonlinear behavior was introduced at strains of $1 \times 10^{-8}$ for Fontainebleau sandstone and $1 \times 10^{-7}$ for Berea sandstone [9]. The thresholds where the conditioning effects occur for the same materials were proposed to be at $5 \times 10^{-7}$ for Berea sandstone, and $2 \times 10^{-7}$ for Fontainebleau sandstone. In our normal experiments, an upper limit of the granite threshold is at $5 \times$ $10^{-7}$. It is probably lower, as the low amplitude measurements take several minutes to perform after having been exposed to the high strain. During this time, the material has moved towards a state equivalent to a lower strain, but not yet to the steady state for the low amplitude it is being exposed to.
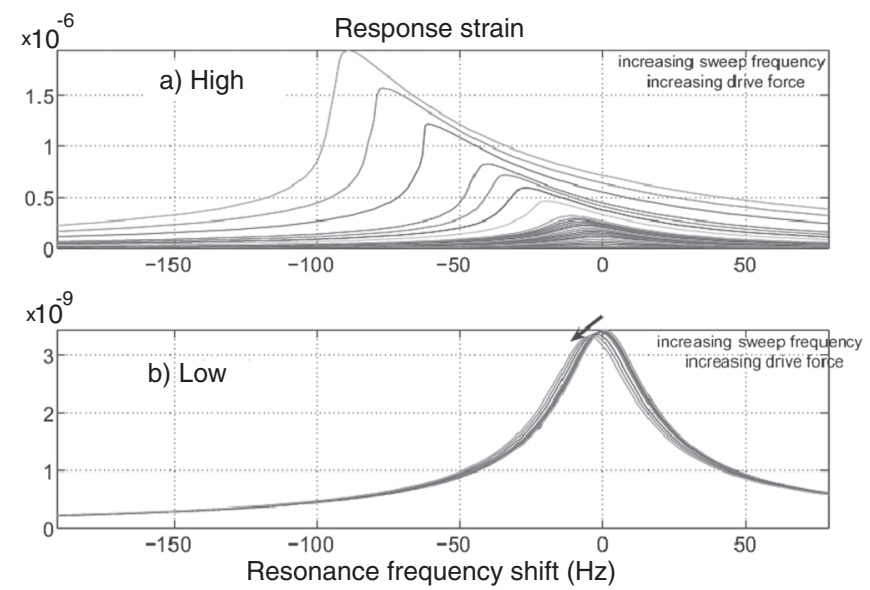

FIG. 5. Dual normal frequency sweeps. They alternate between: (a) high (increasing after every sweep) amplitude frequency sweeps; and (b) low (constant) amplitude normal sweeps. The strain response amplitude is recorded. The low strain initial frequency at shift 0 is at $5346 \mathrm{~Hz}$.

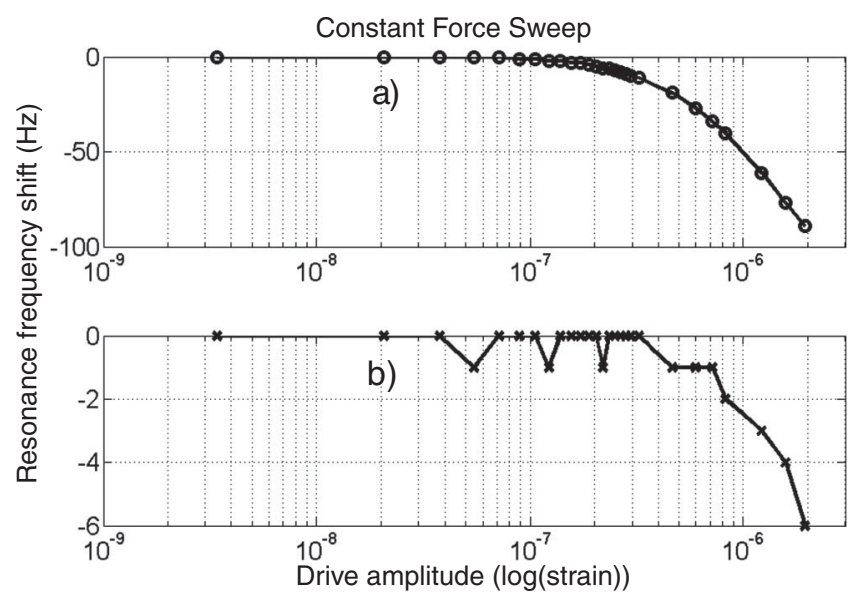

FIG. 6. The resonance frequencies from Fig. 5. In (a), the high (increasing after every sweep) amplitude resonance frequency. In (b), the low (constant) amplitude resonance frequency.

These results are now compared to the curves measured with CSS technique. The curves at the bottom in the Fig. 7 are at the low strain, and the top curves are at the high strains.

The minima representing the resonance frequencies are plotted in Fig. 8. The upper limit of the conditioning threshold is at $8 \times 10^{-7}$. The CSS curves for our granite sample begins to shift at a strain of $1.5 \times 10^{-7}$, and the low amplitude curves starts to shift at a strain of $8 \times 10^{-7}$.

By introducing a constant internal strain state of the measured granite rock, the resonance shift is delayed compared to the normal CFS sweep technique. The SD effect has been minimized, and the nonlinearity is the dominating factor in these results.

In Fig. 9, the high amplitude CFS curve from Fig. 6(a) (curve $A$ ) is shown in a linear plot together with the high

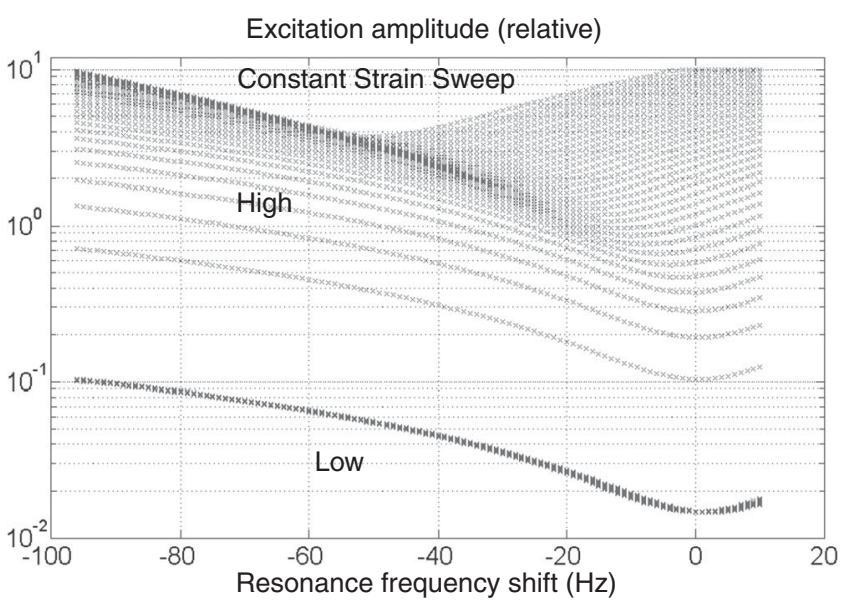

FIG. 7. A dual constant strain frequency sweep. It alternates between high (increasing after every run) amplitude frequency sweeps and constant low strain sweeps. The low strain initial frequency at shift $0 \mathrm{~Hz}$ is at $5265 \mathrm{~Hz}$. 


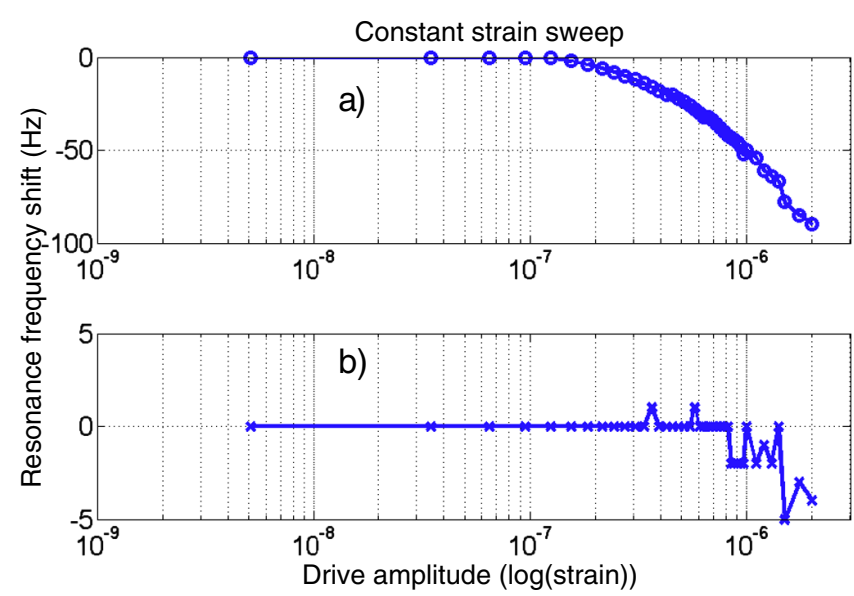

FIG. 8 (color online). The resonance frequencies from Fig. 7. In (a), the high (increasing after every run) amplitude resonance frequency. In (b), the low (constant) amplitude resonance frequency.

amplitude constant strain curves from Fig. 8(a) (curve $B$ ). The curve A contains the simultaneous influence of the slow dynamic conditioning and nonlinearity. Every point in curve $B$ contains the nonlinearity at a specific steady thermodynamic state. For every point, that state is different as the strain is different. What has been removed is the change of the state during each sweep. The Slow Dynamics has been minimized.

In curve $C$, the resonance frequency difference between curves $A$ and $B$ is plotted. This is the Slow Dynamics - the influence of the conditioning taking place during the normal sweep in $A$. The slow dynamics and the nonlinearity have been separated. Out of the three parameters: (1) the nonlinearity; (2) the thermodynamic strain level; and (3) the change in time of the thermodynamic strain level (the Slow Dynamics), the third has been removed. This is a considerable improvement, but even now, the physical interpretation is not evident. From the curves in Fig. 9, a few observations can be made. The resonance frequencies for the normal and constant strain are differing strongly at the lowest strain. The resonance frequency is increasing with strain between some points on the nonlinear curve $B$. The Slow Dynamics influence is slowing the process down-because curve $A$ has higher values than curve $B$. The rate of slow dynamics increases with strain, as seen in curve $C$.

In conclusion, the measurement on the steady-state material, at constant strain, shows a different nonlinear dependence than the normal sweeps. This is a measurement of the true nonlinear distortion at each given strain level, where the slow dynamics nonequilibrium dynamics is

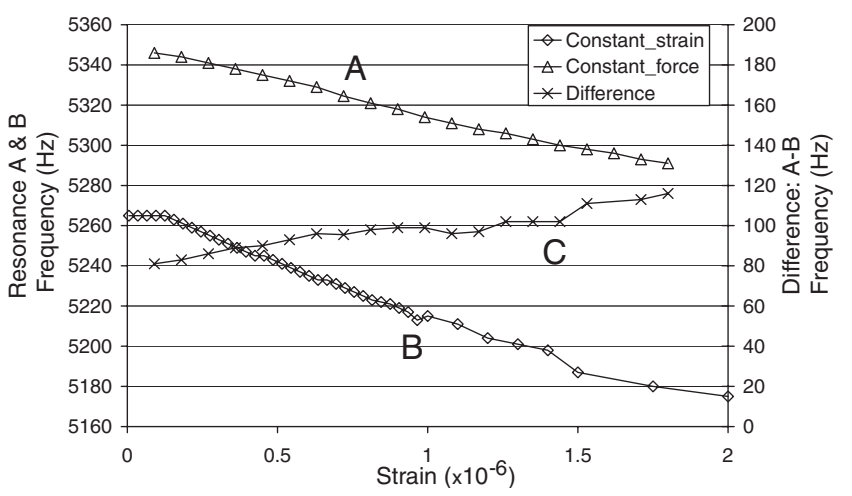

FIG. 9. Comparison between the resonance frequencies, the normal, and the constant strain frequency sweeps as a function of strain amplitude.

minimized. These measurements will together with other existing and upcoming data form the base for discovering the underlying causes behind the nonlinear distortion, the thermodynamic material state properties, and the slow nonequilibrium dynamic processes taking place in rocks and many other materials.

This work is financed by VINNOVA, Sweden.

[1] P. A. Johnson, Materials world : the Journal of the Institute of Materials 7, 544 (1999).

[2] D. Pasqualini, K. Heitman, J.A. TenCate, S. Habib, D. Higdon, and P. A. Johnson, J. Geophys. Res. 112, B01204 (2007).

[3] R. A. Guyer and P. A. Johnson, Phys. Today 52, No. 4, 30 (1999).

[4] J. A. TenCate, E. Smith, and R. A. Guyer, Phys. Rev. Lett. 85, 1020 (2000).

[5] L. A. Ostrovsky and P. A. Johnson, Riv. Nuovo Cimento 24, 1 (2001).

[6] J. A. TenCate and T. J. Shankland, Geophys. Res. Lett. 23, 3019 (1996).

[7] P. A. Johnson and A. Sutin, J. Acoust. Soc. Am. 117, 124 (2005).

[8] K.C.E. Haller and C.M. Hedberg, in Innovation in Nonlinear Accoustics: ISNA 17, edited by A. A. Atchley, V. W. Sparrow, and R. M. Keolia, AIP Conf. Proc. No. 838 (AIP, Melville, New York, 2006), p. 87.

[9] J. A. TenCate, D. Pasqualini, S. Habib, K. Heitmann, D. Higdon, and P. Johnson, Phys. Rev. Lett. 93, 065501 (2004).

[10] K. C.E. Haller and C.M. Hedberg, Proc. 9th Wespac (Acoustical Society of Korea, Seoul, 2006).

[11] R. A. Guyer, J. A. TenCate, and P. A. Johnson, Phys. Rev. Lett. 82, 3280 (1999). 